\title{
Prostate ultrasound: back in business!
}

\author{
Nicolae Crișan ${ }^{1,2}$, Iulia Andraș ${ }^{1,2}$, Corina Radu ${ }^{3,4}$, David Andraș ${ }^{5}$, Radu-Tudor Coman ${ }^{6}$, \\ Paul Tucan ${ }^{7}$, Doina Pîslă ${ }^{7}$, Dana Crișan ${ }^{8,9}$, Ioan Coman ${ }^{1,2}$
}

${ }^{1}$ Urology Department, Clinical Municipal Hospital, ${ }^{2}$ Urology Department, "Iuliu Hatieganu” University of Medicine and Pharmacy, ${ }^{3}$ Internal Medicine Department, "Iuliu Hatieganu" University of Medicine and Pharmacy, ${ }^{4}$ "Octavian Fodor" Regional Institute of Gastroenterology and Hepatology, ${ }^{5}$ Oncologic Surgery Department, "Ion Chiricuta" Institute of Oncology, ${ }^{6}$ Epidemiology Department, "Iuliu Hatieganu" University of Medicine and Pharmacy, ${ }^{7} \mathrm{Re}-$ search Center for Industrial Robots Simulation and Testing, Technical University, ${ }^{8}$ Internal Medicine Department, $5^{\text {th }}$ Medical Clinic, "Iuliu Hatieganu" University of Medicine and Pharmacy, ${ }^{9}$ Internal Medicine Department, Clinical Municipal Hospital, Cluj-Napoca, Romania

\begin{abstract}
The use of grey scale prostate ultrasound decreased after the implementation of magnetic resonance imaging (MRI) for the diagnosis and evaluation of prostate cancer. The new developments, such as multiparametric ultrasound and MRI-ultrasound fusion technology, renewed the interest for this imaging method in the assessment of prostate cancer. The purpose of this paper was to review the current role of prostate ultrasound in the setting of these new applications. A thorough reevaluation of the selection criteria of the patients is required to assess which patients would benefit from multiparametric ultrasound, who would benefit from multiparametric MRI or the combination of both to assist prostate biopsy in order to ensure the balance between overdiagnosis and underdiagnosis of prostate cancer.
\end{abstract}

Keywords: prostatic neoplasms; image-guided biopsy; magnetic resonance imaging; ultrasonography

\section{Introduction}

The diagnosis of prostate cancer $(\mathrm{PCa})$ using transrectal greyscale ultrasound (US) is limited only to the identification of the hypoechoic lesions in the peripheral zone of the prostate, which account for $50-70 \%$ of the $\mathrm{PCa}$ cases [1]. A normal US does not necessarily mean that PCa is absent, but the lack of visibility on US is usually associated with a low grade cancer. On the other hand, the biopsy of a suspect lesion has a two times higher likelihood of diagnosing clinically significant $\mathrm{PCa}$ (high volume and grade) [2].

Received 16.07.2017 Accepted 25.09.2017

Med Ultrason

2017, Vol. 19, No 4, 423-429

Corresponding author: Iulia Andraș, $\mathrm{MD}, \mathrm{PhD}$

Clinical Municipal Hospital,

Department of Urology

11 Tabacarilor, 400139, Cluj-Napoca, Romania

Phone: +40 744374996

E-mail: dr.iuliaandras@gmail.com
Systematic US-guided prostate biopsy is performed in a randomized manner according to the European Association of Urology Guidelines [3], which leads to the underdiagnosis of an important rate of clinically significant $\mathrm{PCa}$ [4]. In this setting, magnetic resonance imaging (MRI) has emerged as a new standard for the diagnostic evaluation and staging of prostate cancer [5]. Prostate MRI assesses four sequences: T2 weighted imaging (T2WI), dynamic contrast enhancement (DCE-MRI), diffusion weighted imaging (DWI-MRI) and spectroscopy. As a result, the use of T2-WI in combination with at least two functional techniques (DCE, DWI, spectroscopy) is referred to as multiparametric MRI (mpMRI). The anatomical sequence (T2-WI) is used for the detection of prostate cancer, whereas the functional techniques offer information regarding the perfusion and metabolic profile of the lesion [6].

Aiming to standardize the report and techniques for mpMRI, the European Society of Urogenital Radiology introduced the Prostate Imaging Reporting and Data 
System (PI-RADS), which was recently updated and is used to estimate the risk for the presence of clinically significant cancer ( 1 - very low; 5 - very high) [7]. Vargas et al showed that PI-RADS version 2 can diagnose up to $95 \%$ of prostate cancer foci with a volume higher than $0.5 \mathrm{ml}$ [8]. Other authors confirmed the importance of MRI in the diagnosis of prostate cancer and reported a negative predictive value for mpMRI of $97 \%$ for the presence of aggressive prostate cancer [9]. Furthermore, the PROMIS trial has shown that the use of mpMRI as a triage test at the first presentation might avoid $27 \%$ of prostate biopsies [10].

Although these results seem very promising, there is significant variability in the published studies [11], depending of the number of cases and the experience of the radiologist. Lower volume centers report a negative predictive value of mpMRI as low as $63 \%$ [12] and a moderate rate of interreader agreement, even after the implementation of PI-RADS version 2 (kappa=0.478) [13].

Furthermore, there are intrinsic limitations of $\mathrm{mp}$ MRI. Approximately $80 \%$ of tumors smaller than $0.5 \mathrm{ml}$ are missed by mpMRI, even though they are clinically significant (Gleason score $\geq 4+3$ ) [5] and tumor foci $\geq 0.5$ $\mathrm{ml}$ that contain Gleason 4 with a cribriform pattern remain undiagnosed in $64 \%$ of cases [14].

Although mpMRI has shown multiple advantages for the diagnosis and characterization of prostate cancer, its use in everyday clinical practice is still limited and the results are impaired by the differences in MRI protocols and reporting, and the experience and training of the radiologists.

As a result, the new US techniques that can be used for the assessment of prostate disease (contrast-enhanced ultrasound and elastography) and the new role of ultrasound in association with MRI for the guidance of prostate biopsy (cognitive and fusion) restore its importance for the diagnosis of prostate cancer.

The present paper aims to review the current role of US for the diagnosis of $\mathrm{PCa}$ and prostate biopsy.

\section{Conventional transrectal grey scale ultrasound}

Prostate greyscale US has a low sensitivity and specificity for the detection of $\mathrm{PCa}$. The suspicion is raised by the presence of a hypoechoic area, but this appearance is characteristic only for about $60 \%$ of PCa. The rest of PCa nodules are isoechoic, therefore they are usually missedat US $[15,16]$. Furthermore, only $17-57 \%$ of the hypoechoic areas at greyscale US are diagnosed as malignant at biopsy [17].

Prostate US has an important role in performing prostate biopsy by guiding the needle towards the peripheral zone of the prostate, but the low sensitivity in detection of the malignant nodules does not always allow a targeted biopsy. As a result, the standard prostate biopsy is performed in a randomized manner. A number of technical aids have been developed in order to improve the detection rate of $\mathrm{PCa}$ and ensure a targeted biopsy: color and power Doppler US, contrast-enhanced US (CEUS), elastography, multiparametric US, and magnetic resonance imaging.

\section{New ultrasound techniques}

\section{Color and power Doppler US}

Color and power Doppler US allow the assessment of tumor angiogenesis. It is considered that the microvascular density is high in neoplasms and is associated with a high grading and a negative prognosis. On the other hand, benign conditions that associate enhanced vascularity, like infection or inflammation, might lead to a false positive result. However, studies have shown that color Doppler can increase the $\mathrm{PCa}$ detection rate of grey scale US with $12 \%$ [18].

Power Doppler ultrasound is a more sensitive examination, allowing the identification of blood vessels of 1 $\mathrm{mm}$. But the tumor angiogenesis vessels are much smaller, with a caliber of 10-15 microM, thus limiting their identification [19-20].

Still, a number of studies have demonstrated an increase from $47 \%$ to $74 \%$ of the specificity of grey scale prostate US by using power Doppler [21].

Furthermore, Sauvain et al showed that power Doppler US can predict the presence of high-risk PCa. The authors concluded that patients with a normal power Doppler transrectal US have less than 5\% risk of aggressive $\mathrm{PCa}$, in cases of prostate specific antigen $<10 \mathrm{ng} / \mathrm{ml}$ and normal digital rectal examination [22].

\section{Contrast-enhanced ultrasound}

CEUS uses intravenous gas-filled microbubbles of similar size with erythrocytes, thus allowing an efficient exploration of the microvessels. CEUS improves the detection rate of $\mathrm{PCa}$ up to $86 \%$ [23], but can also assist targeted prostate biopsy, increasing the number of positive biopsy cores in comparison with systematic biopsy $(11 \%$ vs $5 \%)$ [24].

In a comparative study between CEUS and MRI, which used as a reference the prostatectomy specimen pathological analysis, sensitivity of $58-69 \%$ and specificity of $93-95 \%$ of CEUS for the detection of lesions $>0.5$ $\mathrm{ml}$, similar to MRI [25] was demonstrated. Furthermore, Qi et al [26] observed that transrectal CEUS combined with grey scale US has a detection rate of index lesion of $80.7 \%$ in comparison with $66 \%$ for contrast-enhanced MRI [27]. 
A recent study brought in the assessment of new parameters for CEUS - the dispersion and velocity of the US contrast agent, which can improve the detection rate of PCa by a better assessment of prostate vascularization [28].Another recently developed technique is the threesection contrast-enhanced transrectal US, which showed sensitivity of $92.3 \%$, specificity of $69.2 \%$, and accuracy of $78.1 \%$ for the detection of PCa. The higher rate of low grade $\mathrm{PCa}$ detected allowed the selection of the patients for active surveillance [29].

Due to the fact that CEUS is highly user-dependent, a new technique has been developed in order to translate the subtle malignancy signs from CEUS recordings into parametric maps, based on a statistical analysis of the wash-in rate. Postema et al observed that the use of CEUS parametric maps to target the prostate biopsy allows decreasing the number of biopsy cores performed with more that $70 \%$, with only $8.5 \%$ rate of missed clinically significant cancers [30].

\section{Elastography}

Elastography allows the assessment of prostate tissue rigidity and the differentiation between soft and hard areas inside the prostate $[31,32]$. A harder area is the result of an increased cellularity, microvessels, and collagen deposits [33]. The majority of false positive results are caused by prostatitis and hyperplastic nodules [33].

A prospective unicentric study [34] included 109 patients with PCa assessed by elastography before undergoing radical prostatectomy. The radiologist recorded the suspect areas (right lobe, left lobe, base, middle, apex), which were compared with the prostatectomy specimen pathological analysis (3 $\mathrm{mm}$ sections). The elastography identified 439 suspect areas in comparison with the pathological examination, which identified 451 malignant areas. The sensitivity and specificity of elastography for the detection of $\mathrm{PCa}$ were $75.4 \%$ and $76.7 \%$ respectively, with a positive predictive value of $87.8 \%$, negative predictive value of $59 \%$, and accuracy of $76 \%$. For lesions $>5 \mathrm{~mm}$ and Gleason score $\geq 7$, elastography showed a specificity of over $80 \%$ [34].

Another possibility is to use elastography for the targeting of prostate biopsy. A meta-analysis of 6 studies, published in 2012, showed a sensitivity of $62 \%$ and a specificity of $79 \%$ for the diagnosis of PCa using elastography-targeted biopsy, with better results for patients with higher Gleason scores [34-36].

Currently, there are two elastographic techiques used for endorectal prostate assessment: strain elastography (SE) and, more recently, novel shear wave elastography (SWE).

$\mathrm{SE}$ requires a manual compressions and decompressions upon the prostate tissue with the endorectal probe. This technique has demonstrated advantages for the de- tection of $\mathrm{PCa}$, but also for the guidance of prostate biopsy [37]. Brock et al [38] assessed SE in association with MRI-US fusion prostate biopsy in patients with a history of negative biopsy, but persistent suspicion of PCa. The authors observed that elastography significantly increased the prediction of PCa at biopsy (AUC 0.86 vs AUC 0.79). Furthermore, the quantification of SE into a strain index (calculated as the ratio between the region of interest corresponding to a lesion and to a normal area in the prostate) has been shown to correlate with the Gleason score in malignant prostate lesions [37].

In SWE technique the transducer automatically generates the acoustic radiation impulse. This type of elastography has the advantage of providing quantitative information regarding the tissue stiffness and deformation, with no difference in the intra-observer reproducibility [39]. The two studies published so far that compared SWE with sextant prostate biopsy demonstrated high sensitivity and specificity for the diagnosis of PCa $(96 \%$ and $95 \%$ in the first study $90 \%$ and $80 \%$ in the second study) [40,41].

\section{Multiparametric ultrasound}

Multiparametric US is a new concept defined as the combination of CEUS and real-time elastography. The combination of these two techniques was demonstrated to decrease the false-positive results and increase the positive predictive value for the presence of $\mathrm{PCa}$ [42]

Brock et al assessed $100 \mathrm{PCa}$ patients before undergoing radical prostatectomy and the suspicious areas were marked into 12 sectors (every prostatic lobe was split in anterior, posterior, base, middle, apex). The results were compared with the pathological result of the prostatectomy specimen by analyzing $4 \mu \mathrm{m}$ sections. The positive predictive value of multiparametric USfor the detection of PCa was $89.7 \%$. The main advantage of CEUS was to reduce the rate of false positive results of real-time elastography with two-thirds [42].

Another retrospective study demonstrated a detection rate of PCa for multiparametric US of 59.4\% (56.5\% for elastography, $74.2 \%$ for CEUS). These results were obtained using a median of 5 biopsy cores/patient and a median of 3 cores/lesion. The highest detection rate was for patients with prostate volume $<40 \mathrm{ml}(72.2 \%)$ and patients older than 70 years $(87 \%)$ [43].

\section{Ultrasound-MRI-guided prostate biopsy}

Two methods have been developed in which ultrasound and mpMRI can be combined in order to assist prostate biopsy: cognitive and fusion biopsy.

MRI-US guided cognitive prostate biopsy

MRI-US guided prostate biopsy implies that the MRI scan of the patient is performed prior to biopsy, with the 
identification of suspicious lesions by the radiologist. In order to improve the communication with the urologist, the radiologist can use several methods: delineation of the lesion directly on the MRI images, employment of the sector map of PI-RADS version 2 [7] or localization of the lesion using landmarks like bladder neck, cysts, or hyperplastic nodules [44]. Prostate biopsy is performed with US guidance and the lesion is targeted based on the aforementioned details. Lai et al concluded that, in the setting of a previous negative biopsy, performing MRIUS guided cognitive prostate biopsy and sampling only the suspicious lesions can increase $\mathrm{PCa}$ detection rate up to $20.8 \%$ in comparison with $14 \%$ for a systematic USguided repeat biopsy [44].

\section{MRI-US fusion guided prostate biopsy}

MRI-US fusion guided prostate biopsy is based also on a prior MRI scan. It implies the use of a fusion software that overlaps the MRI of the prostate with the transrectal US in real-time. The lesion is marked on the MRI of the prostate and, after the fusion of the two volumes - MRI and US - the software transposes the lesion mark onto the US, enabling the targeted biopsy. Lacetera et al reported their first experience with MRI-US fusion guided prostate biopsy and showed a clinically significant $\mathrm{PCa}$ detection rate of $40 \%$ in the setting of repeat biopsy [45].

A recent systematic review by Wegelin et al [46] showed that MRI guided biopsy (any type) is not superior to US guided prostate biopsy in overall cancer detection, but shows improvement in the detection of clinically significant prostate cancer (MRI guided biopsy missed 10\%, whereas US guided biopsy missed $21 \%$ clinically significant cancers). Furthermore, fusion guided biopsy did not show significant advantages over cognitive biopsy in terms of overall cancer detection $(\mathrm{p}=0.11)$ or clinically significant prostate cancer detection $(\mathrm{p}=0.62)$.

MRI-US guided prostate biopsy is a complex technique that faces several limitations in the practical use $[47,48]$. Apart from the already mentioned MRI limitations, there are also biopsy-related limitations, such as the software registration type or the costs of the procedure.

One of the most important factors to ensure correct localization of the lesion during fusion biopsy is the software registration type. For performing the fusion between the two volumes, elastic systems take into consideration prostate movements and deformation during the insertion of the US probe, whereas rigid systems relay only on internal anatomical markers [49]. Delongchamps et al [50] showed that elastic image fusion was more accurate for cancer detection than rigid image fusion, which performed similarly with cognitive guided biopsy [51].
Incorporation of MRI and MRI-guided prostate biopsy is not without costs. Although Cerantola et al [52] developed a model that suggests that the integration of MRI for assisting the diagnosis and biopsy of prostate cancer is a cost-effective measure even at 20 years after initial diagnosis, the authors admit that the study has some limitations due to the fact that they did not consider the costs related to complications associated with treatment or active surveillance. Furthermore, van de Ven et al [53] showed that almost two thirds of the lesions with a PIRADS score of 5 on MRI were also visible on transrectal US, thus questioning the real need to increase the costs using MRI guided biopsy of these lesions.

Another question that rises is whether systematic biopsy is still needed in addition to MRI guided biopsy. Salami et al [54] showed that performing only MRI targeted biopsy in patients with highly suspicious lesions still misses $3.5 \%$ of clinically significant prostate cancers, and $6.2 \%$ of the patients that undergo fusion biopsy are upgraded to clinically significant disease at concurrent systematic biopsy [55].

\section{Discussions}

It is clear nowadays that conventional transrectal US has reduced its importance in the diagnostic assessment of PCa. But its availability, costs, and the possibility to repeat the examination whenever necessary make it an asset for the urologist. A number of techniques have been developed in order to improve the sensitivity and specificity of grey scale US and they have proven their advantages in terms of $\mathrm{PCa}$ detection rate and the guidance of prostate biopsy [15]. Multiparametric MRI has revolutionized the diagnostics of $\mathrm{PCa}$ and offered the possibility to perform a true targeted biopsy, but the costs of the investigation, the low volume centers, and the lack of specialists hinder its implementation [12].

A concern that was raised regarding $\mathrm{PCa}$ is the problem of overdiagnosis of clinically indolent tumors and underdiagnosis of aggressive $\mathrm{PCa}$ [3]. Since the majority of clinically significant $\mathrm{PCa}$ is visible using grey scale and new US techniques [2], probably a complex multiparametric US approach would be the best to be offered when patients present with PCa suspicion. In the setting of one negative biopsy and persistent suspicion for $\mathrm{PCa}$, the high accuracy of multiparametric MRI in the identification of $\mathrm{PCa}$ [9] makes this investigation the standard for the evaluation of the prostate gland and targeting of the biopsy.

A combination of both techniques might be of help to not miss clinically significant PCa in patients at risk for his malignancy and at least one negative prostate biopsy. 
Last, but not least, another issue that was much debated in the last years is whether it is possible to decrease the number of biopsy cores for diagnosis, with the purpose of decreasing the associated morbidity of the procedure. Despite having a high positive and negative predictive value for the presence of PCa, MRI-guided prostate biopsy cannot completely replace systematic biopsy so far [54]. But, as new US techniques develop and multiparametric MRI becomes increasingly used worldwide, the premises are that systematic biopsy will soon be forgotten.

In conclusion, although the use of prostate US has decreased after the implementation of MRI, the new developments in US technique have renewed the interest for this imaging method for the assessment of prostate cancer. Power-Doppler, CEUS, multiparametric US, and elastography have demonstrated an increase in the diagnosis of significant PCa. More complex techniques such as MRIUS guided biopsy increase even more the accuracy of prostate biopsy, but still face some limitations in terms of technical issues and costs. A thorough reevaluation of the selection criteria of the patients is required to assess which patients would benefit from multiparametric US, which would benefit from mpMRI or the combination of both to assist prostate biopsy in order to ensure the balance between overdiagnosis and underdiagnosis of PCa.

Acknowledgements: This paper was realized within the Partnership Programme PN-II, which runs with the financial support of MEN-UEFISCDI, Project no. $247 / 2014$

\section{Conflict of interest: none}

\section{References}

1. Ghai S, Toi A. Role of transrectal ultrasonography in prostate cancer. Radiol Clin North Am 2012;50:1061-1073.

2. Toi A, Neill MG, Lockwood GA, Sweet JM, Tammsalu LA, Fleshner NE. The continuing importance of transrectal ultrasound identification of prostatic lesions. J Urol 2007;177:516-520.

3. Mottet N, Bellmunt J, Bolla M, et al. EAU-ESTRO-SIOG Guidelines on Prostate Cancer. Part 1: Screening, diagnosis, and local treatment with curative intent. Eur Urol 2017;71:618-629.

4. Mohler JL, Armstrong AJ, Bahnson RR, et al. Prostate cancer, Version 3.2012: featured updates to the NCCN guidelines. J Natl Compr Canc Netw 2012;10:1081-1087.

5. Barentsz JO, Richenberg J, Clements R, et al. ESUR prostate MR guidelines 2012. Eur Radiol 2012;22:746-757.

6. Woodrum DA, Kawashima A, Gorny KR, Mynderse LA. Prostate cancer: state of the art imaging and focal treatment. Clin Radiol 2017;72:665-679.
7. Weinreb JC, Barentsz JO, Choyke PL, et al. PI-RADS Prostate Imaging - Reporting and Data System: 2015, Version 2. Eur Urol 2016;69:16-40.

8. Vargas HA, Hötker AM, Goldman DA, et al. Updated prostate imaging reporting and data system (PIRADS v2) recommendations for the detection of clinically significant prostate cancer using multiparametric MRI: critical evaluation using whole-mount pathology as standard of reference. Eur Radiol 2016;26:1606-1612.

9. Lu AJ, Syed JS, Nguyen KA, et al. Negative Multiparametric Magnetic Resonance Imaging of the prostate predicts absence of clinically significant prostate cancer on 12-core template prostate biopsy. Urology 2017;105:118-122.

10. Ahmed HU, El-Shater Bosaily A, Brown LC, et al. Diagnostic accuracy of multi-parametric MRI and TRUS biopsy in prostate cancer (PROMIS): a paired validating confirmatory study. Lancet 2017;389:815-822.

11. Woo S, Suh CH, Kim SY, Cho JY, Kim SH. The diagnostic performance of Prostate Imaging Reporting and Data System version 2 for detection of prostate cancer: a systematic review and diagnostic meta-analysis. Eur Urol 2017; 72: 177-188.

12. Barentsz J, de Rooij M, Villeirs G, Weinreb J. Prostate Imaging-Reporting and Data System Version 2 and the implementation of high-quality prostate Magnetic Resonance Imaging. Eur Urol 2017;72:189-191.

13. Zhao C, Gao G, Fang D, et al. The efficiency of multiparametric magnetic resonance imaging (mpMRI) using PIRADS Version 2 in the diagnosis of clinically significant prostate cancer. Clin Imaging 2016;40:885-888.

14. Truong M, Hollenberg G, Weinberg E, Messing EM, Miyamoto H, Frye TP. Impact of Gleason subtype on prostate cancer detection using Multiparametric MRI: Correlation with final histopathology. J Urol 2017, doi:10.1016/j. juro.2017.01.077.

15. Pummer K, Rieken M, Augustin H, Gutschi T, Shariat SF. Innovations in diagnostic imaging of localized prostate cancer. World J Urol 2014;32:881-890.

16. Ellis JH, Tempeny C, Sarin MS, Gatsonis C, Rifkin MD, McNeil BJ. MR imaging and sonography of early prostatic cancer: pathologic and imaging features that influence identification and diagnosis. Am J Roentgenol 1994;162:865872.

17. Seitz M, Scher B, Scherr M, et al. Imaging procedures to diagnose prostate cancer. Urologe A 2007;46:W1435-W1446.

18. Amiel GE, Slawin KM. Newer modalities of ultrasound imaging and treatment of the prostate. Urol Clin North Am 2006;33:329-337.

19. Nelson ED, Slotoroff CB, Gomella LG, Halpern EJ. Targeted biopsy of the prostate: the impact of color Doppler imaging and elastography on prostate cancer detection and Gleason score. Urology 2007;70:1136-1140.

20. Russo G, Mischi M, Scheepens W, De la Rosette JJ, Wijkstra H. Angiogenesis in prostate cancer: onset, progression and imaging. BJU Int 2012;110:E794-E808.

21. Eisenberg ML, Cowan JE, Carroll PR, Shinohara K. The adjunctive use of power Doppler imaging in the preopera- 
tive assessment of prostate cancer. BJU Int 2010;105:12371241.

22. Sauvain JL, Sauvain E, Papavero R, Louis D, Rohmer P. Limiting overdiagnosis of low-risk prostate cancer through an evaluation of the predictive value of transrectal and power Doppler ultrasonography. J Ultrasound 2016;19:275-280.

23. Sedelaar JPM, van Leenders GJ, Goossen TE, et al. Value of contrast ultrasonography in the detection of significant prostate cancer: correlation with radical prostatectomy specimens. Prostate 2002;53:246-253.

24. Mitterberger MJ, Aigner F, Horninger W, et al. Comparative efficiency of contrast-enhanced colour Doppler ultrasound targeted versus systematic biopsy for prostate cancer detection. Eur Radiol 2010;20:2791-2796.

25. Postema A, Mischi M, de la Roette J, Wijkstra H. Multiparametric ultrasound in the detection of prostate cancer: a systematic review. World J Urol 2015;33:1651-1659.

26. Qi T, Chen Y, Zhu Y, Jiang J, Wang L, Qi J. Contrast-enhanced transrectal ultrasonography for detection and localization of prostate index tumor: correlation with radical prostatectomy findings. Urology 2014;84:138-143.

27. Puech P, Potiron E, Lemaitre L, et al. Dynamic contrastenhanced magnetic resonance imaging evaluation of intraprostatic prostate cancer: correlation with radical prostatectomy specimens. Urology 2009;74:1094-1099.

28. van Sloun RJ, Demi L, Postema AW, de la Rosette JJ, Wijkstra H, Mischi M. Ultrasound-contrast-agent dispersion and velocity imaging for prostate cancer localization. Med Image Anal 2017;35:610-619.

29. Qi TY, Sun HG, Li NF, Feng H, Ding YL, Wang XX. Value of three-section contrast-enhanced transrectal ultrasonography in the detection of prostate cancer. J Clin Ultrasound 2017;45:304-309.

30. Postema AW, Frinking PJ, Smeenge M, et al. Dynamic contrast-enhanced ultrasound parametric imaging for the detection of prostate cancer. BJU Int 2016;117:598-603.

31. Giurgiu CR, Manea C, Crişan N, Bungărdean C, Coman I, Dudea SM. Real-time sonoelastography in the diagnosis of prostate cancer. Med Ultrason 2011;13:5-9.

32. Dudea SM, Giurgiu CR, Dumitriu D, et al. Value of ultrasound elastography in the diagnosis and management of prostate carcinoma. Med Ultrason 2011;13:45-53.

33. Zhang Y, Tang J, Li YM, et al. Differentiation of prostate cancer from benign lesions using strain index of transrectal real-time tissue elastography. Eur J Radiol 2012;81:857862.

34. Salomon G, Köllerman J, Thederan I, et al. Evaluation of prostate cancer detection with ultrasound real-time elastography: a comparison with step section pathological analysis after radical prostatectomy. Eur Urol 2008;54:1354-1362.

35. Zhang B, Ma X, Zhan W, et al. Real-time elastography in the diagnosis of patients suspected of having prostate cancer: a meta-analysis. Ultrasound Med Biol 2014;40:14001407.

36. Teng J, Chen M, Gao Y, Yao Y, Chen L, Xu D. Transrectal sonoelastography in the detection of prostate cancers: a meta-analysis. BJU Int 2012;110:E614-E620.
37. Hwang SI, Lee HJ, Lee SE, Hong SK, Byun SS, Choe G. Elastographic strain index in the evaluation of focal lesions detected with transrectal sonography of the prostate gland. J Ultrasound Med 2016;35:899-904.

38. Brock M, Löppenberg B, Roghmann F, et al. Impact of real-time elastography on magnetic resonance imaging/ultrasound fusion guided biopsy in patients with prior negative prostate biopsies. J Urol 2015;193:1191-1197.

39. Sang L, Wang XM, Xu DY, Cai YF. Accuracy of shear wave elastography for the diagnosis of prostate cancer: A meta-analysis. Sci Rep 2017;7:1949.

40. Barr RG, Memo R, Schaub CR. Shear wave ultrasound elastography of the prostate: initial results. Ultrasound Q 2012;28:13-20.

41. Ahmad S, Cao R, Varghese T, Bidaut L, Nabi G. Transrectal quantitative shear wave elastography in the detection and characterisation of prostate cancer. Surg Endosc 2013;27:3280-3287.

42. Brock M, Eggert T, Palisaar RJ, et al. Multiparametric ultrasound of the prostate: adding contrast enhanced ultrasound to real-time elastography to detect histopathologically confirmed cancer. J Urol 2013;189:93-98.

43. Aigner F, Schäfer G, Steiner E, et al. Value of enhanced transrectal ultrasound targeted biopsy for prostate cancer diagnosis: a retrospective data analysis. World J Urol 2012;30:341-346.

44. Lai WJ, Wang HK, Liu HT, et al. Cognitive MRI-TRUS fusion-targeted prostate biopsy according to PI-RADS classification in patients with prior negative systematic biopsy results. J Chin Med Assoc 2016;79:618-624.

45. Lacetera V, Cervelli B, Cicetti A, et al. MRI/US fusion prostate biopsy: Our initial experience. Arch Ital Urol Androl 2016;88:296-299.

46. Wegelin O, van Melick HH, Hooft L, et al. Comparing three different techniques for Magnetic Resonance Imagingtargeted prostate biopsies: a systematic review of in-bore versus Magnetic Resonance Imaging-transrectal ultrasound fusion versus cognitive registration. Is there a preferred technique? Eur Urol 2017;71:517-531.

47. Valerio M, Donaldson I, Emberton M, et al. Detection of clinically significant prostate cancer using magnetic resonance imaging-ultrasound fusion targeted biopsy: a systematic review. Eur Urol 2015;68:8-19.

48. Giannarini G, Crestani A, Rossanese M, Ficarra V. Multiparametric Magnetic Resonance Imaging targeted biopsy for early detection of prostate cancer: all that glitters is not gold! Eur Urol 2017;71:904-906.

49. Westhoff N, Siegel FP, Hausmann D, et al. Precision of $\mathrm{MRI} /$ ultrasound-fusion biopsy in prostate cancer diagnosis: an ex vivo comparison of alternative biopsy techniques on prostate phantoms. World J Urol 2017;35:1015-1022.

50. Delongchamps NB, Peyromaure M, Schull A, et al. Prebiopsy magnetic resonance imaging and prostate cancer detection: comparison of random and targeted biopsies. J Urol 2013;189:493-499.

51. Puech P, Rouvière $\mathrm{O}$, Renard-Penna R, et al. Prostate cancer diagnosis: multiparametric MR-targeted biopsy with cognitive and transrectal US-MR fusion guidance versus 
systematic biopsy--prospective multicenter study. Radiology 2013;268:461-469.

52. Cerantola Y, Dragomir A, Tanguay S, Bladou F, Aprikian A, Kassouf W. Cost-effectiveness of multiparametric magnetic resonance imaging and targeted biopsy in diagnosing prostate cancer. Urol Oncol 2016;34:119.e1-9.

53. van de Ven WJ, Sedelaar JP, van der Leest MM, et al. Visibility of prostate cancer on transrectal ultrasound during fusion with multiparametric magnetic resonance imaging for biopsy. Clin Imaging 2016;40:745-750.
54. Salami SS, Ben-Levi E, Yaskiv O, et al. In patients with a previous negative prostate biopsy and a suspicious lesion on magnetic resonance imaging, is a 12-core biopsy still necessary in addition to a targeted biopsy? BJU Int 2015; 115:562-570.

55. Muthigi A, George AK, Sidana A, et al. Missing the mark: prostate cancer upgrading by systematic biopsy over Magnetic Resonance Imaging/Transrectal ultrasound fusion biopsy. J Urol 2017;197:327-334. 